\title{
Per l'edizione scientifica digitale dei Documenti d'Amore di Francesco da Barberino: modelli, metodi e strumenti
}

\begin{abstract}
Questo saggio è dedicato al lavoro compiuto per la realizzazione dell'edizione digitale dei Documenti d'Amore di Francesco da Barberino. Un'edizione che pone diverse questioni relative alla critica del testo digitale nell'ambito dei testi manoscritti medievali. La riflessione affronta diversi componenti dell'edizione (trascrizione TEI, rete semantica, facsimile) che si intrecciano e si legano nella struttura testuale attraverso la fase di modellazione.

This paper aims to highlight the work carried out for the realisation of the digital scholarly edition of the Documenta Amoris by Francesco da Barberino: an edition project that raises several issues concerning digital textual scholarship in the context of medieval manuscripts. The analysis deals with the different digital components of the edition (TEI transcription, semantic Web technologies, digital facsimile) that intertwine and bind in the textual structure through the process of modelling.
\end{abstract}

Parole chiave: Edizione scientifica digitale, facsimile, codifica, filologia digitale, Linked Open Data, Web semantico

\section{Di cosa parliamo: l'edizione scientifica digitale}

Questo saggio presenta il lavoro, ancora in progress, svolto per la realizzazione dell'edizione digitale dei Documenti d'amore di Francesco da Barberino. La prassi editoriale, ripensata in modo completamente sganciato dalla tecnologia a stampa, ha consentito di elaborare un nuovo modello di rappresentazione testuale di quest'opera e di creare uno spazio di scambio e interconnessione fra campi disciplinari differenti. Nel corso del contributo verrà messo in rilievo l'impianto metodologico e teorico da cui proviene questo progetto digitale, ricostruendo l'assetto sulla base 
dell'orizzonte epistemologico riguardante le cosiddette edizioni scientifiche digitali ${ }^{1}$ al fine di indagare i principi e i metodi della critica testuale digitale nell'ambito dei testi manoscritti medievali.

Il processo di rimediazione, ${ }^{2}$ ossia la capacità di tradurre le forme e i contenuti da una vecchia tecnologia a nuove modalità comunicative, è un passaggio tutt'altro che neutro. Ne è la prova il terreno esegetico sul quale viene tradito il testo dei Documenti d'amore, le cui soluzioni editoriali a stampa, di cui accenneremo più avanti, costringono l'impianto dell'opera a versioni del testo che sollevano non poche problematiche. L'evoluzione tecnologica ha prodotto cambiamenti sostanziali riguardo alle pratiche filologiche: i linguaggi formali entrano inevitabilmente in gioco nella costruzione delle strutture filologiche e semiologiche del testo interagendo come nuovi parametri di interpretazione e fruizione dell'opera. Se partiamo dall'assunto che un'edizione digitale può «arricchire la lettura», ${ }^{3}$ offrendo l'opportunità di superare le limitazioni e l'immobilità del testo guthenberghiano, possiamo allora andare a ricercare significati e significazioni nell'impatto che lo strumento stesso determina nella produzione e nella ricezione del testo costituendone in molti casi il carattere della comunicazione e del messaggio medesimo. ${ }^{4}$

L'edizione dei Documenti d'Amore si inserisce all'interno di un dibattito molto ampio sull'ecdotica computazionale ${ }^{5}$ e digitale. Negli ultimi venti anni, la critica testuale digitale si è sviluppata sotto la pressione dell'evoluzione repentina della tecnologia e del Web, di nuovi strumenti a disposizione e dell'obsolescenza incalzante; in questo contesto, il lavoro del filologo (digitale) viene sollecitato a sperimentare e trasformare le forme editoriali e trovare soluzioni per strutture e forme del testo, e modelli computazionali che meglio si adattano all'interpretazione pensata dall'editore.

Questo progetto è una ulteriore sfida che la critica testuale accoglie inserendosi nel dibattito riguardante le nuove tecniche editoriali e all'interno di un ulteriore cambiamento epistemologico delle edizioni scientifiche digitali. L'edizione digitale dei Documenti d'Amore ha le caratteristiche di un laboratorio sperimen-

\footnotetext{
1 Con questo termine si vuole definire un campo d'indagine sviluppatosi intorno all'ecdotica digitale. Alcuni riferimenti bibliografici: Mancinelli, Pierazzo, Che cos'è un'edizione scientifica digitale; Robinson, What is a Critical Digital Edition?; Pierazzo, Digital scholarly editing; Sahle, What is a Scholarly Digital Edition?.

2 Cfr. Bolter, Lo spazio dello scrivere.

3 Sahle, What is a digital scholarly edition, p. 21.

4 Cfr. McLuhan, La galassia Gutenberg.

5 Cfr. Mordenti, Appunti per una semiotica della trascrizione nella procedura ecdotica computazionale.
} 
tale non solo per ciò che concerne le tecnologie ma anche per una riflessione sulle stesse possibilità di rappresentazione testuale di un'opera manoscritta complessa come quella di Barberino. L'evoluzione della tecnologia, infatti, negli ultimi venti anni ha alterato e reso più complesse molte delle fasi della filiera del lavoro editoriale ${ }^{6}$ centralizzando sempre di più il ruolo dell'editore digitale evolvendo i modelli, le metodologie e i metodi editoriali tipici della prima fase del Web e attraverso l'avvento delle tecnologie del Web semantico, come architetto dell'informazione e di organizzazione della conoscenza. Il cambiamento di paradigma coinvolge una varietà di forme di presentazione del testo, di tecnologie e strumenti impiegati, ${ }^{7}$ e di modelli di rappresentazione. Un testo, di qualunque genere esso sia, non si presenta mai nella sua nudità, ma è sempre accompagnato dal paratesto, ovvero da una serie di elementi ausiliari, che hanno un ruolo importantissimo poiché presentano il testo, nel senso corrente del termine, ma anche per renderlo presente al mondo, assicurarne la ricezione e la diffusione. Secondo Gérard Genette, ${ }^{8}$ la seconda tipologia di trascendenza testuale è costituita da quegli elementi che formano l'opera letteraria (titolo, prefazione, etc.); il paratesto in un'edizione digitale entra a far parte del sistema testuale anche nella stratificazione di linguaggi formali, di metadati, risorse esterne collegate al progetto editoriale e infrastrutture tecnologiche che divengono imprescindibili per l'oggetto digitale; infatti, come vedremo più avanti, molti sono i dati per l'edizione dei Documenti d'Amore interconnessi a risorse esterne.

Patrick Sahle afferma che una delle differenze fra edizione a stampa e edizione digitale sia proprio il fatto che un'edizione digitale «non può essere stampata senza una perdita di informazioni e/o funzionalità». ${ }^{9}$ Affermazione tanto più vera in un contesto come quello del Web 3.0 che concentra l'attenzione sui dati e non sul documento, come avveniva invece con il Web 1.0.

Nonostante le inconfutabili differenze, il concetto di edizione critica digitale come sistema informativo è molto vicino alle riflessioni teoriche e metodologiche della critica testuale, dei metodi consolidati dell'ecdotica e delle teorie legate alla semiotica. Questo assunto viene ribadito dalle parole che Contini utilizza in un saggio su Bédier, secondo cui l’edizione critica «[è] come ogni atto

6 Cfr. Pierazzo, Digital Scholarly Editing: Theories, Models and Methods.

7 Sono stati creati diversi cataloghi in linea per raggruppare le edizioni scientifiche digitali. Un catalogo particolarmente aggiornato e rigoroso è quello a cura di Patrick Sahle (http:// www.digitale-edition.de/) oppure il catalogo curato da Greta Franzini che raccoglie in modo meno selettivo i progetti di edizioni digitali - Catalogue of Digital Editions (https://dig-ed-cat. acdh.oeaw.ac.at); ultimo accesso: maggio 2021.

8 Cfr. Genette, Palinsesti.

9 Sahle, What is a digital scholarly edition, p. 27. 
scientifico, una mera ipotesi di lavoro, la più soddisfacente (ossia economica) che colleghi in sistema i dati». ${ }^{10}$ Un'edizione scientifica digitale, e soprattutto un'edizione digitale semantica, ${ }^{11}$ è un sistema molteplice e variegato di elementi e di relazioni che possono essere formalizzate attraverso asserti, aggiungendo contenuto semantico ai dati e stabilendo connessioni a vari livelli fra questi. A ciò bisogna aggiungere che un testo è legato ai modi della trasmissione e della ricezione, e di conseguenza, inevitabilmente, alle forme della sua rappresentazione e presentazione: «si può ben dire che ogni testo ponga un proprio problema - più e meno complesso - di accertamento filologico e di presentazione al lettore [. . .]. Un'edizione critica è appunto il tentativo di risolvere questo problema». ${ }^{12}$

Quello di cui si terrà conto nelle pagine seguenti è proprio questo modelloparatesto digitale e computazionale che ha contribuito in modo essenziale a ripensare l'opera di Barberino come un oggetto dinamico, non statico, una risorsa che interconnette e elabora dati e che dialoga e crea interazione con altre risorse contenute e organizzate nell'universo di Internet e, in particolare, del Web.

Verranno descritti gli obiettivi raggiunti in questa prima fase mettendo in luce anche i passaggi di tale processo e sottolineando che l'introduzione di tecnologie diverse dalla semplice codifica ha comportato una serie di problemi teorici e metodologici da una parte, e di soluzioni sperimentate ad hoc per l'assenza di strumenti di editing e di pubblicazione dall'altra, conseguentemente facendo scaturire nuovi aspetti teorici. Un'edizione digitale può essere modificata sia occasionalmente, per correggere errori e simili, sia sistematicamente, per aggiungere nuovi testi, applicativi, commenti, voci bibliografiche, etc. Una data di pubblicazione non implica, infatti, la fine del processo di redazione, ma solo l'inizio di una nuova fase. ${ }^{13} \mathrm{La}$ tecnologia ci impone un continuo aggiornamento non solo delle competenze tecniche ma anche dell'affermazione di nuovi modelli e processi e anche dei problemi di sostenibilità.

10 Contini, Ricordo di Joseph Bédier, p. 369.

11 Qualche contributo che ha affrontato il ruolo del semantic Web con la filologia digitale: Cfr. Francesca Tomasi, L'edizione digitale e la rappresentazione della conoscenza e Daquino, Giovannetti, Tomasi, Linked Data per le edizioni scientifiche digitali.

12 Inglese, Come si legge un'edizione critica, p. 16.

13 Cfr. Pierazzo, Digital Scholarly Editing: Theories, Models and Methods. 


\section{Strutture testuali e modelli ermeneutici}

La prima fase di realizzazione di un'edizione digitale è caratterizzata dalla consueta attività tradizionale della recensio, che si riflette nella definizione degli obiettivi, nell'esame del materiale e, non ultimo, nella definizione di un modello formale e astratto su cui poi, successivamente, basare e scegliere la tecnologia. L'operazione più semplice che si può compiere - o, almeno, così sembrerebbe «per guardare da Gutenberg a von Neumann, è quella di prendere un testo [. . .] e memorizzarlo. Questa operazione [. . . ] modifica lo statuto del testo». ${ }^{14} \mathrm{Si}$ tratta quindi di ripensare l'opera all'interno di nuovo modello interpretativo e di testualità e di pianificare il progetto anche sulla base della selezione di dati già digitalizzati e quelli non ancora in formato digitale. I Documenti d'Amore fanno emergere diverse questioni: un testo che viene tràdito da due manoscritti conservati alla Biblioteca Apostolica Vaticana, il Barb. lat. 4077, lasciato incompiuto, e il Barb. lat. 4076, un codice che trasmette il testo nella sua interezza; quest'ultimo, vede Francesco da Barberino impegnato per sedici anni come «architetto e ingegnere insieme»: ${ }^{15}$ un progetto letterario autografo, ambizioso e complesso, con una struttura ideata a priori, e dettagliatamente elaborata.

Il macrotesto e le lingue utilizzate sono elementi che si riflettono nell'architettura dello spazio del manoscritto coinvolgendo non solo la disposizione delle entità testuali della mise en page ma gli stessi aspetti paleografici e anche iconografici. La lirica volgare viene posta al centro, la traduzione in prosa e il commento in latino ai lati, a corredo dei quali si pone la componente iconografica, dando vita a un contesto testuale copioso di rimandi e allegorie: «le tre componenti [. . . ] svolgono funzioni comunicative differenti ma compongono nello stesso tempo un insieme fortemente coeso, caratterizzato dalla 'circolarità' del discorso e delle possibilità di lettura». ${ }^{16}$ Le edizioni moderne sottolineano chiaramente i limiti della tecnologia a stampa nella rappresentazione di un'opera densa di elementi eterogenei su un supporto «materiale-sensoriale» ${ }^{17}$ come i Documenti d'Amore: l'edizione curata da Egidi, ${ }^{18}$ pubblicata nel 1901, e la più recente pubblicata nel 2008, curata da Albertazzi, ${ }^{19}$ evidenziano chiaramente come il cambiamento del supporto comporti un problema di fruizione e trasmissione dell'intera opera.

14 Gigliozzi, Introduzione all'uso del computer negli studi letterari, p. 181.

15 Goldin Folena, Il commento di Francesco Da Barberino, p. 274.

16 Panzera, Francesco da Barberino tra Andrea Cappellano e Averroè, p. 40.

17 Mordenti, Informatica e critica dei testi, p. 60.

18 Francesco da Barberino, I Documenti d'Amore (ed. Egidi).

19 Francesco da Barberino, I Documenti d'Amore / Documenta amoris (ed. Albertazzi). 
Il testo dell'edizione semidiplomatica curata da Egidi è il punto di partenza del progetto di edizione digitale, sia per l'accuratezza del testo offerto dal curatore, sia perché si dispone di una versione in formato .pdf del libro (originariamente a stampa) che permette di utilizzare software di riconoscimento automatico della scrittura - OCR (Optical Character Recognition) - per acquisire il testo su cui lavo-

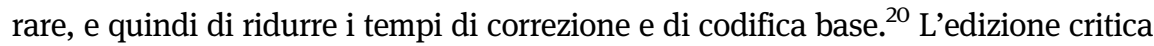
di Egidi è fondata sul codice autografo Barb. lat. 4076, composto da 102 carte; un testo tuttavia lacunoso, integrato, previa collazione, con il manoscritto Barb. lat. 4077, rimasto a sua volta incompleto. Gli obiettivi prefissati, in questa prima fase, sono quindi la correzione del testo dell'edizione di Egidi, la descrizione delle aree del manoscritto collegate alla trascrizione e delle caratteristiche che appartengono al documento nel suo contesto materiale e l'annotazione delle strutture testuali dei Documenti d'Amore. L'edizione digitale nel modello dei metadati e nelle informazioni contenute, non è soltanto un testo univoco, ma un ipotesto che può contenere in sé varie trascrizioni e includere obiettivi diversi, più ampi e complessi. Infatti, il testo in un ambiente virtuale, a differenza di quello cartaceo, può non soltanto cambiare velocemente nel tempo ma può anche generare all'interno di uno stesso progetto digitale diversi risultati alla questione 'quale edizione per quale lettore' - diverse edizioni, in modo potenzialmente infinito, non solo nella presentazione interattiva ma anche nel modello di metadati. In questa prima fase, la scelta di trascrivere il testo di Egidi collegandolo alle aree del manoscritto è dunque, per i suddetti motivi, meramente strumentale e legata a un problema di ordine pratico. Questi dati come vedremo più avanti saranno descritti in un sistema di annotazioni che permette di disambiguare e distinguere i livelli testuali e anche di garantire attribuzioni di informazioni relative alle edizioni di Egidi e di Albertazzi.

\section{Trascrizione e modellizzazione}

Una delle attività di ricerca alla base della filologia digitale, e dell'informatica umanistica più in generale, è la costruzione di modelli interpretativi dell'oggetto di studio che si vuole rappresentare. In fondo anche la critica testuale o l'ecdotica si basano tradizionalmente su procedure e metodi legati a strutture interpretative e livelli testuali (paratestuali, metatestuali o intertestuale, etc.)

20 Nonostante ci siano diversi software sviluppati per il riconoscimento di scritture particolari, la cosiddetta HTR (Handwriting Text Recognition), non ci è sembrato opportuno per ragioni di tempo sperimentare in questa fase. 
che esprimono il punto di vista del critico e dell'editore sul testo e sul suo contesto. Possiamo riconoscere per quanto concerne i tradizionali studi umanistici molti modelli o strutture più o meno esplicite: pensiamo, ad esempio, alla struttura di una voce di un dizionario o all'apparato critico; questi sono schemi codificati a cui si fa riferimento senza tuttavia un livello di precisione tale da sostenere la creazione di un modello formale e di dati strutturati. Il testo come sistema complesso può essere scomposto in unità segniche, selezionate e riorganizzate a loro volta in un modello formale e coerente di dati (formalizzando anche le relazioni fra questi). La "modellazione" consiste proprio nell'identificazione delle componenti testuali, che tradotte e trasposte in metadati e in altri tipi di linguaggi formali, compongono un ulteriore paratesto dell'edizione digitale:«modellare significa ragionare sia sulle componenti logiche interne del testo, che sulla rete delle relazioni [. . .]. Questi sono i dati che andranno formalizzati attraverso gli adeguati strumenti concettuali per estrarre nuova conoscenza». ${ }^{21}$ La critica del testo vede nel modello dei dati e nel processo di modellazione una componente fondamentale dell'editing digitale in cui vengono esplicitate e tradotte le ipotesi interpretative del curatore in soluzioni formali.

La costruzione del modello è essenziale nella sua fase iniziale, durante la recensio, ma coinvolge allo stesso modo anche i successivi momenti della filiera editoriale. Aspetti della critica testuale digitale e aspetti tecnologici sono stati oggetto della prima fase dell'edizione dei Documenti d'Amore; nello specifico la riflessione sul secondo aspetto, e cioè quella sul dato tecnologico, ha creato un rallentamento nelle fasi di lavoro. Il progetto si è concentrato su quattro elementi in particolare: la struttura e la trascrizione del testo, il collegamento di questo al manoscritto, il ruolo del facsimile e l'indice dei nomi menzionati.

La trascrizione è il punto di partenza di un'architettura del testo più complessa, "proprio perché il momento della trascrizione appare decisivo» ${ }^{22}$ nelle procedure dell'ecdotica digitale. I fondamenti teorici a cui ci riferiamo vengono avanzati da alcuni filologi interessati alle trasformazioni del testo attraverso la tecnologia già a partire dagli anni Ottanta ${ }^{23}$ ma poi sviluppatesi altresì nelle più recenti riflessioni sul Web semantico ${ }^{24}$ e sulle "forme del testo digitale". Raul Mordenti sottolinea come l'applicazione delle tecnologie vada a influenzare e modificare maggiormente quelle che nel paradigma precedente erano le fasi più

21 Tomasi, L'informazione digitale e il Web semantico, p. 164.

22 Mordenti, Informatica e critica dei testi, p. 56.

23 Da ricordare i contributi della cosiddetta scuola romana che vede come protagonisti Giuseppe Gigliozzi, Raul Mordenti, Tito Orlandi.

24 In particolare i lavori e le questioni metodologiche affrontate da Fabio Ciotti e Francesca Tomasi. 
meccaniche, ossia da un lato la trascrizione del testo, e dall'altro la pubblicazione dell'edizione. La centralità di tale argomento è legata alla costruzione dell'intero impianto del modello di edizione dei Documenti d'Amore. Per la struttura base del testo e alcuni aspetti della trascrizione è stato scelto come linguaggio di marcatura lo standard TEI (Text Encoding Initiative). Utilizzato in molti progetti di edizioni e archivi digitali, ${ }^{25}$ il progetto TEI è volto allo sviluppo di un modello di codifica standard che ha come obiettivo la rappresentazione e la gestione dei dati umanistico-letterari in ambiente digitale. Nell'edizione dei Documenti d'Amore l'annotazione ha caratterizzato la descrizione degli aspetti della trascrizione vertendo in particolare sulle suddivisioni delle tipologie testuali appartenenti al testo. La TEI è un metalinguaggio basato su XML - una specifica pubblicata dalla W3C, ovvero un consorzio per i linguaggi standard del Web - e che dal 1987 stabilisce e implementa un vocabolario comune per la descrizione di archivi di dati per umanisti attraverso standard di uno specifico dominio concettuale. L'XML ha una sintassi con una struttura ad albero che, applicata a una certa idea di testo, può tuttavia essere molto limitante.

Due sono gli elementi fondamentali della trascrizione in questa prima fase: la descrizione della struttura testuale con l'individuazione delle tipologie testuali e la trascrizione del testo con una codifica base dell'edizione Egidi. Il passo successivo sarà poi quello di creare una nuova edizione critica considerando e confrontando il testo dei due manoscritti con la rappresentazione formale di un nuovo apparato critico che comprenderà anche le immagini facsimilari dei codici. Il modello dei Documenti d'Amore attraverso la codifica TEI viene suddiviso in cinque tipologie testuali secondo la struttura pensata da Barberino: la lirica, la traduzione in prosa latina, le glosse latine e le iconografie; queste macro divisioni che attraversano tutto il testo vengono rappresentate dai tag o marcatori $\left.{ }^{26}<\operatorname{div}\right\rangle^{27}$ e un attributo type che ne identifica la categoria. Le unità testuali vengono descritte per la lirica, con i tag $<\mathrm{lg}>$ che rappresenta gruppi di versi e $<\mathrm{l}>$ che identifica i singoli

25 TEI è stato concepito originariamente come un formato di interscambio: è uno schema la cui prima pubblicazione uscita nel 1993 ha riunito diversi studiosi e progetti per consentire una comunicazione sul piano dei metadati di un modello astratto. Il vocabolario della TEI nel suo complesso non è qualcosa di fisso e immobile, ma si è evoluto nel corso degli anni e si sviluppato anche grazie alle svariate esigenze di una comunità con progetti ed esigenze diverse. Uno standard, quindi, creato da elementi testuali e oggetti che rispondono all'insieme di peculiarità significative individuate dai membri dei gruppi di lavoro TEI, ed è stato perfezionato e rivisto nel corso di diversi decenni, durante i quali sono stati aggiunti nuovi oggetti e quelli esistenti sono stati rivisti per coerenza e chiarezza.

26 Con il termine 'tag' si identifica un marcatore di un vocabolario di metadati.

27 ' $<$ div $>$ ' definisce una porzione di testo, una divisione testuale. 
versi, e per i blocchi di testo nella prosa sia volgare sia latina viene utilizzato il tag $<\mathrm{ab}\rangle$. La decisione di non utilizzare il tag $<\mathrm{p}\rangle$ per i paragrafi di Egidi è dovuta al fatto che sarà anche un testo preparatorio per una nuova edizione critica; infatti il tag <ab> è definito nelle linee guida della TEI: «un componente che funge da contenitore anonimo di sintagmi o elementi interlivello simili al paragrafo ma senza il bagaglio semantico di quest'ultimo». ${ }^{28}$

Del testo di Egidi vengono codificate le note a piè di pagina con il tag <note> che includono sia gli interventi critici dell'editore sia l'apparato critico. Queste note vengono distinte con un attributo type per distinguere pertanto l'apparato critico posto in nota dal curatore e anche alcuni marginalia. Ma alcuni di questi vengono inseriti ai lati della pagina dell'edizione e per tale ragione distinguiamo con il marcatore <label> quando sono titoli dell'apparatus e quando sono addizioni del testo (collocati da Egidi in nota). Nel manoscritto si trovano generalmente in inchiostro rosso, o sottolineati sia in rosso che in nero. Per questo motivo al tag <label > viene aggiunto un attributo rend che qualifica e distingue l'inchiostro utilizzato. L'esempio di seguito può esplicitare quanto detto:

$<$ label type="marginalia" rend="red" place="above" $>$ de amoris passione $</$ label $>$

$<$ label type="marginalia" $><$ hi rend="underline" $>$ de custodia $</$ hi $></$ label $>$

Questo modello di codifica indica quindi elementi di testi appartenenti a diverse fonti: vengono inseriti due livelli di interpretazione nella annotazione della trascrizione e viene data attenzione alla formattazione utilizzata dall'autore e dal curatore: da una parte l'utilizzo di un formattazione del corsivo, dall'altra l'utilizzo dell'inchiostro.

Il codice Barb. lat. 4076 comprende poi altri elementi, identificati attraverso ulteriori annotazioni: viene segnalata la presenza dei segni di interpunzione con un tag <pc $>$ e le lacune con il tag <gap > che attraverso l'utilizzo degli attributi distingue la tipologia della lacuna che può comprendere così più righe di testo, come nell'esempio che segue:

<gap reason="illegible" unit="line" quantity="2" /> oppure <gap reason="illegible" unit="line" atLeast="4" atMost="8"/>

28 La definizione dal sito Web della TEI: https://www.tei-c.org/Vault/P5//1.4.1/doc/tei-p5-doc /it/html/ref-ab.html. 
oppure singoli caratteri:

$<$ gap reason="illegible" unit="char" quantity="2" /> <gap reason="illegible" atLeast="4" atMost="8" unit="char"/>

Un altro aspetto della codifica prende in considerazione la divisione della struttura delle [. . . ] pagine (dell'edizione Egidi) o delle carte (dei due testimoni). Attraverso il marcatore $\langle\mathrm{pb}\rangle$ quindi vengono allineati il testo e le immagini dei due codici preservate e pubblicate nel progetto digitale del sito della biblioteca Vaticana e segnalato l'inizio delle pagine del volume di Egidi. L'attributo @ed indica, molto semplicemente, il riferimento al manoscritto che viene ampiamente descritto nella manuscript description nella TeiHeader e come vedremo più avanti nell'ultimo paragrafo a una base di conoscenza esterna che va a descrivere i livelli filologici che spesso nella codifica TEI possono essere confusi o sovrapposti; l'attributo @xml:id qualifica all'elemento un identificatore univoco più complesso e dettagliato:

$<$ pb ed="MSS_Barb.lat.4076" xml:id="BarLa_lir_1r"/>

$<$ pb ed="MSS_Barb.lat.4077" xml:id="BarLa_lir_1r" />

L'edizione Egidi avrà anche il numero delle pagine corrispondenti con un attributo $\mathrm{n}=$ "numero della pagina":

<pb ed="Egidi" n="4" xml:id="egidi_lir_00001"/>

\section{Il facsimile}

L'evoluzione del Web ha permesso sempre più la proliferazione di progetti dedicati alla pubblicazione di immagini digitali di documenti del patrimonio culturale, facilitando l'accesso e la diffusione di riproduzioni delle fonti primarie. Ne è scaturito di conseguenza un cambiamento decisivo dell'ecdotica e delle pratiche editoriali nell'ambito digitale: ${ }^{29}$ da una parte, incoraggiando sempre più l'inclusione dei facsimili delle edizioni scientifiche e, dall'altra, contribuendo a un accrescimento dell'aspettativa dei lettori all'accesso all'immagine. ${ }^{30}$ Nel cosiddetto Web $1.0,{ }^{31} \mathrm{le}$

29 Cfr. Pierazzo, Facsimile and Document-Centric Editing.

30 Pierazzo, Digital genetic editions, p. 202.

31 Mosaic, il primo browser che integrava le immagini all'interno del testo, entra in uso nel 1993. Vedi Mancinelli, Pierazzo, Che cos'è un'edizione scientifica digitale. 
immagini venivano spesso evitate (erano infatti incluse all'interno di edizioni o archivi in CD-ROM) perché difficilmente scaricabili e gestibili: la connessione poteva creare ostacoli alla fruibilità della risorsa o dell'intero progetto. Sembra scontato in questo momento, ma il Web non ha sempre dato la possibilità di includere elementi multimediali o multimodali. La disponibilità delle riproduzioni dei facsimili delle fonti documentarie ha cambiato la pratica ecdotica e la testualità incentivando, per esempio, la produzione di edizioni digitali documentarie e a testo unico. ${ }^{32}$ In queste tipologie di edizioni, l'immagine del facsimile ha una specifica funzione di controllo della trascrizione o, anche, di testi manoscritti con una particolare mise en page o testi autografi. Nonostante oggigiorno sia molto più agevole introdurre la fotografia digitale nei progetti editoriali, questa viene ancora per lo più impiegata come elemento accessorio o di ornamento.

Il modello che viene proposto per l'edizione dei Documenti d'Amore ha inoltre come base lo studio dell'impianto progettuale ideato da Francesco da Barberino mediante l'analisi della complessa struttura testuale e iconografica dell'opera e, ulteriormente, il confronto delle riproduzioni digitali dei due codici, Barb. lat. $4076 \mathrm{e}$ 4077. Le annotazioni descrivono i blocchi di testo, le iconografie e l'apparato decorativo ma anche le relazioni che intercorrono tra questi elementi, esplicitando così la particolare composizione del manoscritto ideata e prodotta dall'autore in cui l'interpretazione del testo sembra inscindibile dal suo documento materiale.

Nell'ambito dello studio dei manoscritti medievali, lo scambio interdisciplinare può mettere in relazione livelli di analisi e approcci diversi. Il modello può includere un'analisi di tipo codicologico e paleografico collegandola a quella filologica - elementi che per ragioni legate alla tecnologia a stampa si è sempre considerato come studio parallelo o, anche, collegato ma mai congiunto.

Molti sono i progetti che danno rilievo al dominio di ricerca della codicologia e della paleografia ma anche di edizioni di fonti primarie. Indichiamo in particolare tre lavori significativi al riguardo: il primo, DigiPal, ${ }^{33}$ diretto da Peter Stoke, ha l'obiettivo di rappresentare, catalogare e analizzare le scritture medievali nel suo contesto diplomatico e manoscritto, combinando cataloghi digitali, descrizioni di calligrafia e immagini di documenti e delle loro forme di lettere costituenti; il secondo, VisColl, ${ }^{34}$ un progetto che vede la pubblicazione recente della versione 2.0, mira a costruire modelli di dati per la descrizione fisica e la struttura dei manoscritti e la successiva visualizzazione. VisColl, in particolare, verrà utilizzato per la descrizione codicologica dei due manoscritti curata da Sara

32 Cfr. Pierazzo, A rationale of digital documentary editions.

33 DigiPal - http://www.digipal.eu/.

34 VisColl - https://viscoll.org/about/. 
Bischetti e può essere facilmente integrato nel modello dell'edizione dei Documenti d'Amore o in progetti di edizione digitali perché basato in XML/TEI; il terzo, PRISMS, di recentissima pubblicazione, è una piattaforma di Open Scholarship per la realizzazione di edizioni digitali e rappresenta attraverso una rete semantica il materiale digitalizzato delle fonti primarie che, come vedremo più avanti, è molto simile all'approccio impiegato dal Knowledge site ${ }^{35}$ dell'edizione delle Lettere di Vespasiano da Bisticci che abbiamo tenuto in considerazione per il nostro modello.

La varietà di soluzioni tecniche adottate, ideate e implementate ad hoc soprattutto per la presentazione e la pubblicazione di facsimili digitali ha spesso comportato diverse problematiche sia dal punto di vista dell'obsolescenza degli applicativi o dei software (il carattere dell'obsolescenza intrinseco nella tecnologia: un esempio può essere Flash, un applicativo che è andato in disuso dopo pochi anni e adottato in molti edizioni digitali) e per i diversi formati (tif, jpeg, etc.) utilizzati e varie risoluzione dei dati. Il modello dei Documenti d'Amore si espande e comprende la descrizione semantica della constitutio textus dando un significato ecdotico alla classificazione del materiale e delle procedure applicative del metodo utilizzato. Tre diversi livelli di analisi comprendono lo studio delle fonti primarie attraverso il facsimile digitale: 1) una codicologica (VisColl), 2) descrizione del modello ecdotico attraverso la struttura semantica e a grafo, e 3) l'individuazione della struttura dei blocchi di testo dell'apparato decorativo, apparato critico delle iconografie dei due codici attraverso la TEI.

Se la gestione delle immagini digitali è stata caratterizzata non solo da una varietà di formati, di infrastrutture e strumenti di visualizzazione che hanno avuto spesso un periodo di funzionamento temporaneo, per ovviare a queste e altre problematiche relative allo scambio e al riuso dei dati, è stato creato l'International Image Interoperability Framework (IIIF). ${ }^{36}$ Un framework (come lo definisce il nome stesso), nato da un contesto biblioteconomico, che cambia nuovamente anche il lavoro filologico ad esso collegato e spinge la collaborazione fra archivi e critica testuale. Il IIIF permette lo scambio e l'integrazione delle immagini le cui informazioni sia dell'oggetto fisico sia del facsimile digitale sono fornite mediante un manifesto espresso in formato Json-LD (Linked Data). Dare il giusto valore informativo del facsimile digitale attraverso una descrizione che arricchisca la comprensione del lettore (o dell'utente) riguardo alle caratteristiche materiali del documento - condotta, per esempio, con l'uti-

35 L'edizione digitale delle Lettere di Vespasiano da Bisticci è curata da Francesca Tomasi: https://projects.dharc.unibo.it/vespasiano/.

36 IIIF - iiif.io. 
lizzo di metadati pertinenti - è di fondamentale importanza per la fruizione e la comprensione completa dell'oggetto in esame. L'assunto di partenza è che la tecnologia non è neutra. Pertanto l'organizzazione di questi dati, le infrastrutture e il modo in cui sono strutturati creano una narrazione di tutte le componenti di un progetto digitale: «while such collections might be used to create models that enhance our understanding, those models will not work without an acknowledgement of the narrative». ${ }^{37}$ Nonostante queste criticità, non si escludono i vantaggi di tale dato che certamente migliora le modalità di accessibilità e fruibilità di fonti documentarie finora soltanto immaginate. Un'immagine digitale, però, potrebbe essere fuorviante nella comprensione dell'oggetto fisico e della fruizione virtuale degli aspetti materiali. La Biblioteca Apostolica Vaticana ha adottato il framework IIIF per la pubblicazione delle immagini digitali dei codici conservati nella stessa. Questo ha permesso un importante lavoro per lo sviluppo del modello dell'edizione dei Documenti d'Amore ma ha fatto emergere anche dei limiti. dei limiti. Sembra chiaro da quanto detto finora che dare rilievo al ruolo del facsimile digitale come elemento interpretativo e all'interno dell'apparato esegetico dell'opera sia essenziale per la restituzione del testo. L'immagine che ci offre ad oggi la Vaticana non è di altissima qualità, nonostante la tecnologia IIIF che ne permette la pubblicazione, e il sigillo digitale con cui vengono pubblicati le immagini digitali rende alquanto farraginosa l'analisi paleografica.

Questo, dunque, il modello per l'uso del facsimile (Figura 1):

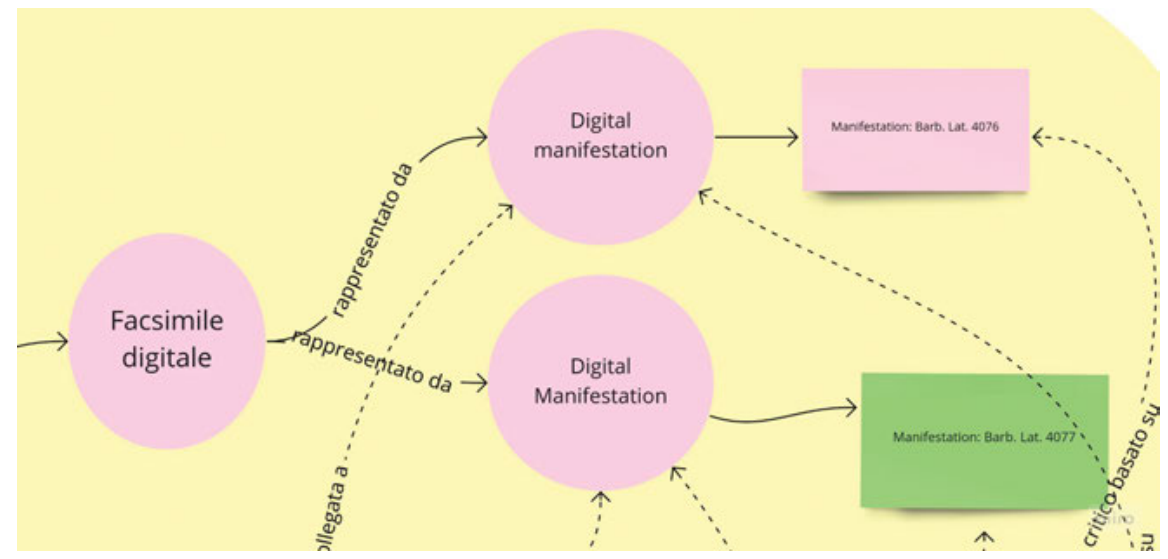

Figura 1: Il modello per la rappresentazione del facsimile.

37 Fafinski, Facsimile narratives, p. 2. 


\section{Un nuovo cambiamento epistemologico}

Il IIIF, sviluppatosi all'interno di una comunità internazionale molto attiva, ha come obiettivo l'interoperabilità ${ }^{38}$ e la condivisione di risorse basate su immagini: ${ }^{39}$ consiste in un ambiente di lavoro che può essere definito come uno «strumento, modello, standard, infrastruttura». ${ }^{40}$ Una serie di API (Application Programming Interfaces $)^{41}$ gestiscono l'architettura che va da componenti server all'esposizione $\mathrm{e}$ alla ricerca. Molte istituzioni bibliotecarie fino a qualche anno fa non consentivano l'utilizzo delle immagini in progetti terzi; al momento, ce ne sono ancora molte (sempre meno) che permettono di consultare immagini, previa autorizzazione, e tramite dispositivi quali CD-ROM; ancora più spesso non si ha l'autorizzazione a riutilizzare quelle risorse. Il IIIF vede la prima versione pubblicata nel 2012 con lo scopo di diffondere i dati preservati dalla stessa istituzione e il loro riutilizzo secondo principi FAIR (Findable Accessible Interoperable Reuse). ${ }^{42}$ Secondo questi principi, i dati della ricerca devono essere risorse da condividere, rendere accessibili e riproducibili ovvero efficacemente riutilizzabili. Il IIIF è una tecnologia nuova e in quanto tale è tuttora soggetta a questioni irrisolte, relative agli strumenti e alle pratiche concrete di impiego, tra cui il modello di rappresentazione delle informazioni relative alle immagini in collegamento con la trascrizione (in TEI), strumenti idonei per manipolare le immagini e per la loro visualizzazione. L'immagine quindi può essere una risorsa esterna ma integrabile e visualizzabile in un altro progetto.

Ma in che modo la riproduzione fotografica può essere funzionale e arricchire il sistema testo di un'edizione digitale? I manoscritti medievali sono di particolare complessità testuale ed estetica e rispondono a funzioni e decisioni di presentazione del libro manoscritto. ${ }^{43}$ Una parte della codifica, infatti, è dedicata ad aspetti grafici e iconografici attraverso la definizione di aree dell'immagine in cui può es-

38 L'interoperabilità è la capacità di un prodotto informatico di scambiare informazioni con altri sistemi.

39 https://iiif.io/model/shared-canvas/1.0/.

40 Dalla documentazione del sito iiif.io.

41 Si tratta di un software intermediario che permette a due applicazioni di comunicare tra loro. Un prodotto o servizio possono parlare con altri prodotti e servizi senza dover sapere come sono implementati. Questo può semplificare lo sviluppo di applicazioni, risparmiando tempo e denaro. Le API adesso a disposizione sono: API Image, API Presentation che permette la descrizione dei metadati in Jason-LD e la API Search, per le funzionalità di ricerca. Queste le più stabili ma ce ne sono altre in versione Beta.

42 La documentazione dei principi FAIR (Findability, Accessibility, Interoperability, Reuse) in inglese può: https://www.go-fair.org/fair-principles/.

43 Cfr. Maniaci, Archeologia del manoscritto. 
sere rappresentato il materiale testuale e iconografico e la struttura stessa del manoscritto. Gli esemplari digitalizzati sono costituiti da numerosi file (o da diverse URL), e nel visualizzare un manoscritto sul computer è necessario ricostruire i vari pezzi di un mosaico. La segnicità materiale dipende dalla tecnologia che lo incorpora: l'immagine di un manoscritto può essere rappresentata, i suoi dati e metadati possono essere processati, scaricati, navigati, ma il codice non può essere toccato né sfogliato. Si tratta dunque di una testualità fluida come realizzazione di azioni che hanno ognuna un valore semantico.

Tutti i componenti esposti di un'edizione scientifica digitale ci restituiscono un quadro molto più che virtuoso di questa complessa architettura; «l'informatica che ci interessa è più un episteme che una tecnologia»: ${ }^{44}$ dati, metadati e elementi relativi alla sostenibilità devono anche essere compresi come un concetto epistemico incorporato in sistemi complessi, non semplicemente come un insieme di problemi da risolvere attraverso da soluzioni informatiche ingegneristiche.

\section{Un dialogo tra tecnologie diverse: IIIF e TEI}

Il IIIF cambia ulteriormente il carattere epistemologico dell'edizione digitale che si afferma come un oggetto dinamico che si interconnette con altre risorse. Questo significa che un'immagine può essere collegata a dei dati scientifici come quelli prodotti dallo studio e dal lavoro che può essere compiuto attraverso la TEI e altre tecnologie del Web semantico. Nonostante la capacità tecnica, il problema che dobbiamo sempre tenere in mente è quale modello per descrivere questa relazione e anche le modalità di flusso di lavoro con immagini in IIIF per il riconoscimento delle aree delle immagini e la codifica compiuta da più editori/codificatori. Se il modello di metadati risponde in senso pratico a un approccio dell'edizione, significherà rappresentare il ruolo del facsimile e la funzione che assume all'interno dell'edizione digitale. Il lavoro editoriale di descrizione delle informazioni sulla riproduzione digitale del testimone Barb. lat. 4076 ha determinato tutto il modello di metadati dei Documenti d'Amore. L'obiettivo è gestire il facsimile e collegare aree dell'immagine alla tipologia di testo. TEI non ha ancora sviluppato standard chiari e condivisi per la descrizione delle informazioni dei documenti per i dati IIIF. Al contempo TEI ha affrontato già con la versione P5 pubblicata nel 2007 il problema della rappresentazione delle risorse delle fonti primarie of-

44 Mordenti, L'altra critica, p. 150. 
frendone una certa varietà di rappresentazione. Il "modulo" 45 indica delle soluzioni per la rappresentazione delle immagini del facsimile, per il collegamento delle immagini, o la rappresentazione di parte delle immagini; le modalità di rappresentazione sono varie: l'elemento <facsimile> contiene l'indicazione di una fonte primaria e quattro sono gli elementi presi in considerazione per la nostra edizione: <facsimile >, <surface $>$, <graphic $>$ e <zone $>$. L'elemento <facsimile $>$ è un elemento che si colloca dopo l'elemento radice $<$ TEI $>$, ed è utilizzato tra la $<$ teiHeader $>$ e l'elemento <text $>$ :

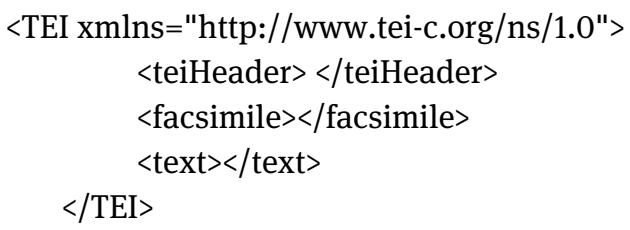

L'elemento <facsimile> contiene l'elemento <surface>, che corrisponde a una carta del manoscritto a sua volta contiene un elemento <graphic $>$ che si collega direttamente alla URL del IIIF attraverso l'attributo @url:

$<$ facsimile $>$

$<$ surface $>$

<graphic url="http://digi.vatlib.it/iiifimage/MSS_Barb. lat.4076/Barb.lat.4076_0018_fa_0004v-5r.jp2/full/full/ 0/default.jpg"/>

Ognuno di essi contiene i riferimenti dei file immagine che rappresentano una carta del manoscritto. La API di IIIF identifica un'immagine e le sue specifiche attraverso una URI veicolata tramite i protocolli HTTP o HTTPS. Questo vuole dire che un client può collegarsi a una immagine disponibile sul Web che il server stesso fornisce con parametri formali espressi esplicitamente della stessa URI secondo la sintassi. Nel nostro caso la URI è quella fornita dalla Biblioteca Vaticana:

http://digi.vatlib.it/iiifimage/MSS_Barb.lat.4076/Barb.lat.4076_0018_fa_ 0004v-5r.jp2/full/full/0/default.jpg

45 Con 'modulo' vengono chiamate le varie sezioni nelle linee guida della TEI riguardanti ognuna un argomento differente: Representation of Primary Source https://www.tei-c.org/release/doc/teip5-doc/en/html/PH.html. 
Abbiamo scelto di non lavorare direttamente con il manifesto IIIF fornito dalla Biblioteca Apostolica Vaticana perché potevamo decidere i valori dei parametri della URI, in cui definire l'area di nostro interesse e creare così un'interpretazione sull'immagine facsimilare. Per organizzare il flusso di lavoro sulle immagini della biblioteca Vaticana in IIIF, e collegare le trascrizioni dei codificatori, dopo aver rifinito il modello di codifica, abbiamo realizzato un software che permettesse il lavoro diretto sulle immagini. ${ }^{46}$ La pianificazione del lavoro di codifica per i collaboratori meno esperti, studenti e dottorandi, è stata infatti pensata per agevolare il lavoro, ed è stato pertanto realizzato un software ad hoc per il progetto Biflow. ${ }^{47}$ Il software è open source, pubblicato sulla piattaforma GitHub, e permette ai codificatori di collegarsi direttamente alle immagini del manoscritto e lavorare sulle stesse tracciando in tal modo le coordinate delle aree interessate. L'elemento $<$ facsimile > può anche essere utilizzato per collegare le iconografie, non porzioni di testo, attribuendo una nota per arricchire la descrizione dell'area annotata. A tale scopo, l'elemento <surface> può contenere uno o più elementi <zone>, ciascuno dei quali rappresenta un'area definita di un'immagine. L'area stessa è delimitata grazie a una serie di attributi sull'elemento $<$ zone $\rangle^{48}$ contenente le coordinate spaziali che permettono di disegnare un'area rettangolare o poligonale. L'elemento <zone> va a indicare un particolare zona che può essere una decorazione o qualsiasi segno che si vuole evidenziare per obiettivi conformi all'edizione oppure può andare a delineare la struttura della pagina, la mise en page inquadrante il testo su tutti e quattro i lati, e in questo caso va a delineare una prassi in uso nel manoscritto giuridico, e in quello universitario a doppio apparato esegetico. Il testo si scompone in testo poetico in volgare al centro della pagina, la traduzione latina ai lati (in prosa), e la glossa che incornicia tutt'intorno entrambi gli strati testuali, ma può essere anche ricomposto a seconda della lettura che l'utente preferisce - a seconda, ovviamente, delle possibilità offerte dalla presentazione. Il software permette di scomporre l'opera e di disegnare le coordinate che serviranno poi a delineare le coordinate corrispondenti a una zona a forma di rettangolo, poligono sopra la stessa immagine. L'attributo che viene utilizzato è point per le coordinate

46 Non è così semplice e ovvio lavorare con immagini IIIF. In rete, sviluppati dalla comunità delle Digital Humanities, si possono trovare degli strumenti per recuperare informazioni di file di immagini (in particolare mi riferisco a lo Zoner sviluppato da Chris Sparks http://teicat. huma-num.fr/zoner.php); questi problemi non permettevano ai codificatori di poter lavorare in modo semplice, collaborativo e veloce. Nel 2020/21 sono stati sviluppati altri strumenti che sono molto simili al 'Barberino generator' (così lo avevamo chiamato).

47 Può essere visitato all'indirizzo Web: https://tmancinelli.github.io/documentidamore/.

48 Il modulo <zone> della specifica della TEI: https://www.tei-c.org/Vault/P5/3.2.0/doc/tei-p5 -doc/en/html/ref-zone.html. 
dell'area, in aggiunta viene corredato da un identificatore unico per utilizzarlo successivamente come risorsa semantica:

<zone points="569,90 679,88 681,250 569,254" xml:id="zone_1v_Recto_Apparatus"/>

Questa operazione viene eseguita grazie ad un meccanismo geometrico familiare, considerando in particolare l'immagine come spazio cartesiano bidimensionale, dove ogni punto può essere definito da due valori, cioè, il valore "ascissa" (o "x") e le coordinate "ordinate" (o "y") di quel particolare valore. Questo permetterà di modellizzare il rapporto tra i fogli di manoscritti, le loro immagini digitali e le trascrizioni di queste carte. Questa informazione potrebbe essere gestita direttamente con il file Json-LD, il manifesto IIIF ma per ora non esiste un vero e proprio modello della TEI per connettere la codifica con il IIIF (o viceversa).

\section{Le edizioni a grafo: gli aspetti semantici dell'edizione digitale}

L'evoluzione delle tecnologie e delle modalità di descrizione dei dati nel Web ha comportato un inevitabile mutamento delle metodologie e nelle procedure di rappresentazione della conoscenza e di conseguenza di un nuovo ripensamento della testualità digitale. Il Web si trasforma sempre di più da un insieme di documenti realizzati per essere letti da utenti - a un Web in cui i dati identificati come risorse assumono un ruolo centrale. Non è una tecnologia dell'ultima ora, lo stesso inventore del web, Tim Berners-Lee, presentò il progetto nel 2001 coniando anche la definizione di "web semantico», ${ }^{49}$ e da allora sono stati sviluppati diversi standard, pubblicati sempre dalla W3C, e modalità di rappresentazione della conoscenza attraverso forme di dati strutturati in ambienti in cui esista un'intercessione semantica fra questi. Per quanto riguarda il panorama dei progetti digitali della letteratura italiana possiamo annoverare tra i primi la collezione delle già ricordate Lettere di Vespasiano da Bisticci, la cui edizione è curata da Francesca Tomasi e dal suo gruppo di ricerca. Tomasi è fra le prime studiose, soprattutto in Italia, a riconsiderare l'edizione digitale sulla base dei principi del Web semantico con lo

49 Berners-Lee, Hendler, Ora, The Semantic Web: A New Form of Web Content That is Meaningful to Computers Will Unleash a Revolution of New Possibilities, 
scopo di creare di «una base di conoscenza». ${ }^{50}$ Le edizioni critiche fino ad ora sono stati progetti il cui grado di interoperabilità è ancora concretamente molto basso e sono difficilmente interconnesse con altre risorse al di fuori del progetto in cui si sviluppano - o riconducibili all'interno di un ambiente semantico in cui vengono "ri-usati" modelli e strutture concettuali astratte.

Nell'edizione dei Documenti d'Amore, la codifica TEI/P5 definisce gli aspetti relativi alla formattazione del testo di Egidi e altri elementi relativi alla trascrizione che non vengono rappresentati in modo semantico. Questo linguaggio di marcatura impone una descrizione del testo con una struttura ad albero che ha evidenziato sin dall'inizio di questo progetto dei limiti per la creazione di un modello articolato e complesso. Il sistema di annotazione dell'edizione dei Documenti d'Amore si struttura in forma di grafo per la rappresentazione di alcuni aspetti per ciò che concerne la critica testuale. Le edizioni digitali possono rendere ancora più esplicito il rapporto con l'evoluzione filologica e la materialità delle fonti che organizzano e presentano, che rimediano ed estendono in modi che permettono nuove forme di accesso. Nell'opera dei Documenti d'Amore il sistema di annotazione semantica e di collegamento con risorse esterne comprende quattro componenti in particolare: l'indice dei nomi e l'indice dei luoghi, la mappa dell'apparato filologico e la descrizione della macrostruttura che divide i documenti in dodici blocchi tematici (Figura 2):

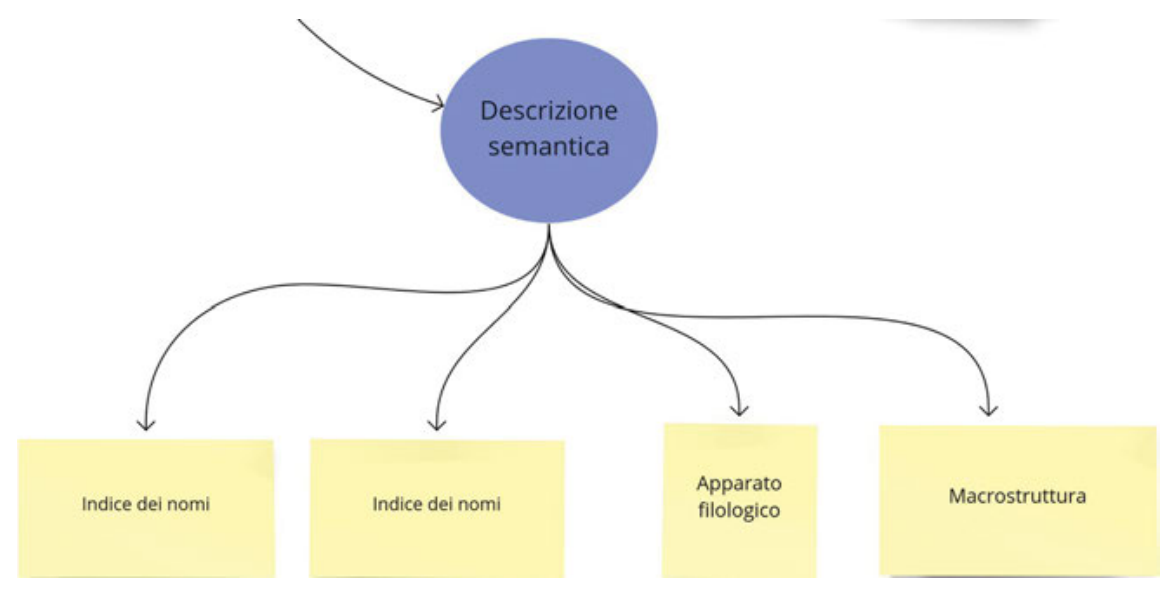

Figura 2: La descrizione semantica della macrostruttura.

50 Tomasi, L'informazione digitale e il Web semantico, p. 286. 
Nella descrizione dell'impianto filologico dell'opera, la descrizione semantica mira a indicare i vari livelli di interpretazione del processo editoriale e le relazioni sui due codici mettendoli a confronto con le due edizioni, vagliando inoltre gli aspetti filologici del testo. Il modello esplicita dunque i riferimenti di critica testuale che compongono l'intera edizione e di cui fanno parte sia la trascrizione sia il facsimile. La trascrizione con alla base il testo di Egidi si collega al facsimile dei due manoscritti: per evitare di incorrere in errori filologici abbiamo deciso di rappresentare l'informazione per esplicitare le funzioni e i ruoli delle quattro fonti (l'edizione curata da Egidi, quella curata da Albertazzi, e i due codici Barb. lat. 4076 e 4077) da cui proviene e si sviluppa questa nuova edizione digitale attraverso le due categorie alla base del modello FRBRoo ${ }^{51}$ chiamate Expression e Manifestation. In questo senso possiamo dichiarare come appartenente a una determinata versione di testo i livelli di responsabilità fra testimoni ed edizioni critiche. Le due categorie utilizzate sono: Espressione con cui si intende «una specifica forma intellettuale o artistica che un'opera assume ogni volta che viene 'realizzata'» ${ }^{52}-$ non la carta o il manoscritto ma l'idea stessa di testo; e Manifestazione con cui si definisce la categoria che rappresenta tutti gli oggetti fisici, sia per quanto riguarda il contenuto intellettuale che la forma fisica. Quindi ogni Manifestation viene esplicitata nel suo uso all'interno della edizione (Figura 3).

Questi concetti sono stati utilizzati anche per il progetto del catalogo Biflow tramite la classe Expression che fa parte di Biflow ontology, ${ }^{53}$ e che si collega a una classe, Manuscript e che basa alcune classi alla ontologia sviluppata per il progetto di Biblissima.

Per ciò che concerne la macrostruttura vengono descritti i dodici documenta ai quali viene assegnata una virtù-allegorica (Docilità, Industria, Costanza, Discrezione, Pazienza, Speranza, Prudenza, Gloria, Giustizia, Innocenza, Gratitudine e Corte d'amore, Eternità) che a loro volta saranno composti da più blocchi di testo che possono essere definite come pars o proemio. Ognuna di queste proprietà punta ai singoli file TEI, considerati in questo particolare caso come 'risorse'. Un altro elemento finora non ancora esplorato è il dominio della lingua. La lingua per il progetto Biflow ha un ruolo cruciale nelle analisi della tradizione manoscritta trecentesca e, ovviamente, per il dominio della traduzione. Simile importanza viene già resa nota da Antonio Montefusco, in un suo intervento sul catalogo Biflow, secondo cui è fondamentale affrontare la questione

51 FRBRoo - http://www.cidoc-crm.org/frbroo/home-0.

52 Vedi la documentazione sul sito ufficiale: FRBRoo - http://www.cidoc-crm.org/frbroo/ home-0; traduzione mia.

53 Ontologia Biflow - https://catalogobiflow.vedph.it/ontology/. 


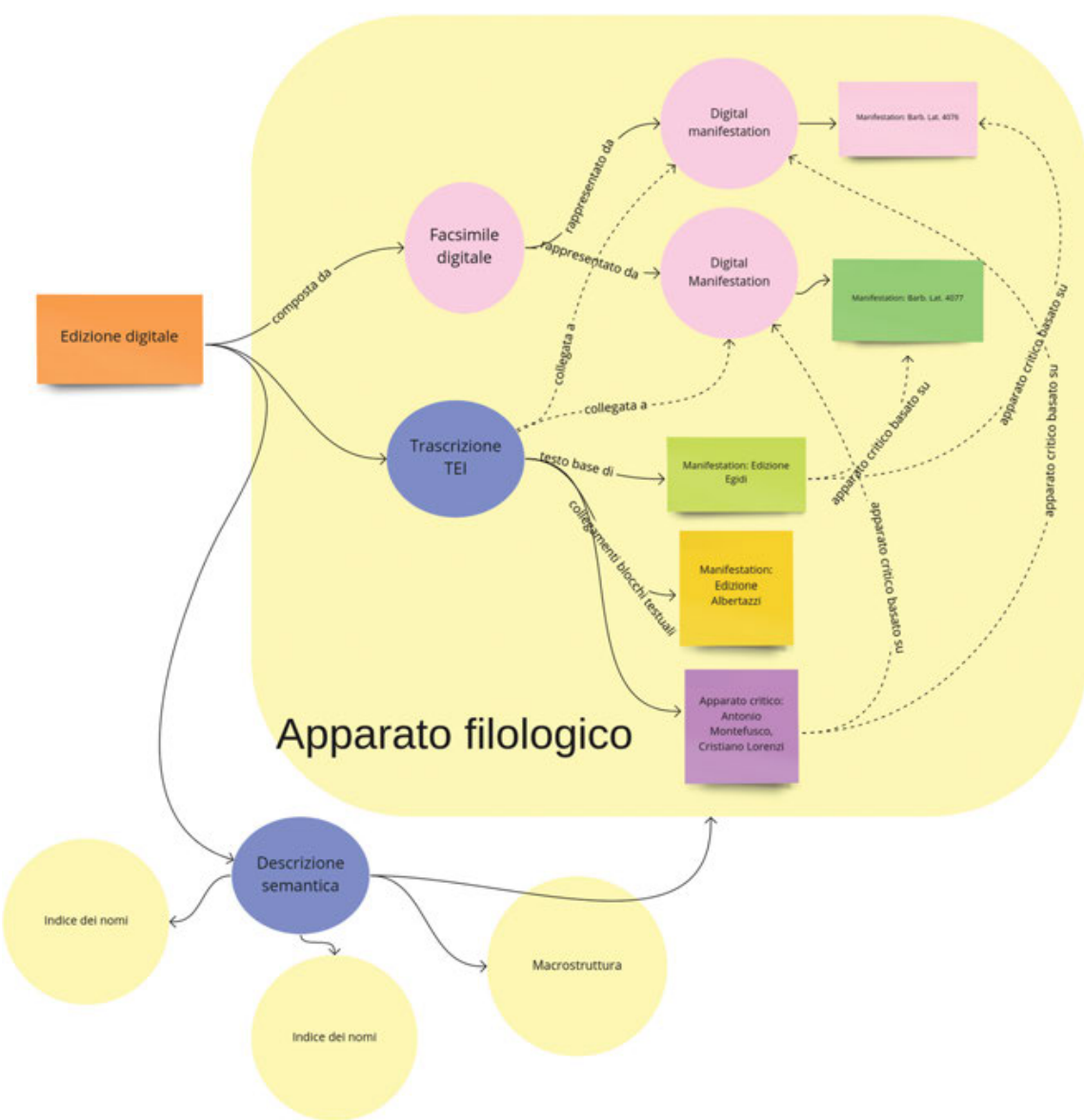

Figura 3: L'apparato filologico descrive semanticamente i file TEl e gli altri elementi dell'edizione.

della lingua «fare storia sociale della lingua, storia sociale del parlare, storia sociale della comunicazione». ${ }^{54} \mathrm{Si}$ tratta di un'importante classe legata alla ontologia Biflow e che può collegare progetti di edizioni scientifiche digitali di un corpus. I file TEI mappano il minimo comune multiplo di queste classi concettuali e ogni blocco di testo si collega (non automaticamente) ai diversi atti interpretativi. Ognuno di questi file TEI è identificato come risorsa ed è referenziato nei documenti semantici RDF per ognuna delle interpretazioni. In questo modo

54 Burke, Lingua, società e storia, p. 7. 
è possibile estrapolare ogni singola porzione dei Documenti d'Amore e comprenderne l'impatto e la collocazione nei contesti interpretativi così da avere chiaro la semantica e gli spettri di analisi.

Vari sono i modelli e varie le ontologie prese in considerazione anche se non rappresentati dal grafico (Fig. C): HiCo, CIDOC-CRM e FRBRoo e, non per ultima, Biflow, l'ontologia del catalogo dei testi bilingue del progetto a cui appartiene l'edizione. Il riuso di categorie e modelli concettuali è una buona pratica da seguire per interscambio e riuso dei dati: i modelli citati sono stati utilizzati perché sono considerati dei domini contigui.

Innanzitutto bisogna andare a individuare una classe definita nel modello ontologico HiCO (sviluppato da Marilena Daquino, Francesca Tomasi e Silvio Peroni), Interpretation act. Questa ontologia ha come obiettivo l'attribuzione e «la descrizione di interpretazioni in senso lato storiche - ovvero che tendono a storicizzare il documento - di cui un editore fornisce una descrizione data-centrica dei contenuti e delle relazioni intrattenute dai soggetti-agenti, che compaiono nel testo pieno del documento». ${ }^{55}$ In questo modello le interpretazioni sono strettamente legate a una nuova opera i cui dettagli sono considerati come fatti. L'interpretation Act ha due proprietà fondamentali: la proprietà hico:hasInterpretationType e hico: hasInterpretationCriterion. La prima stabilisce una classificazione arbitraria dell'interpretazione, che può essere definita semplicemente come filologica, semiotica, linguistica, ecc. ${ }^{56}$ La seconda è una breve spiegazione del criterio utilizzato per dichiarare informazioni estratte da una fonte, ad esempio una trascrizione letterale, un'ipotesi, o l'adozione della letteratura su un argomento specifico.

\section{I Nomi citati e citazioni delle opere: LOD - Linked Open Data}

Un testo come i Documenti d'Amore è strettamente correlato alla vita culturale e politica del tempo, copioso di citazioni di autori e opere coeve. Le relazioni che si intessono e la fonte di ogni testo citato vengono sì codificate e disambiguati attraverso l'utilizzo e il collegamento ad authority file per identificare persone e opere citate. Sono stati scelti i tag della TEI < persname> con un attributo @ref che rimanda a un identificativo univoco e alla descrizione della persona nel tei-

$55 \mathrm{http}: / /$ hico.sourceforge.net/. $56 \mathrm{http} / / /$ hico.sourceforge.net/. 
Header. L'attributo @sameAs collega ai record di DBpedia and Wikidata. Ecco qualche esempio di estrazione della marcatura:

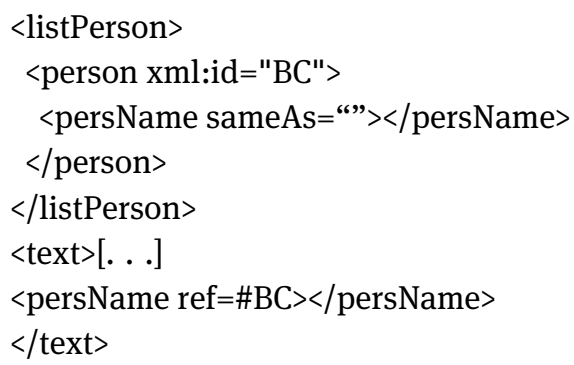

Anche le fonti dei testi citati vengono ulteriormente descritte e disambiguate con un attributo @source che, come per la codifica delle persone, si collega a un identificativo univoco dell'opera citata, a sua volta descritta nel teiHeader e collegata ai record DBpedia e Wikipedia corrispondenti attraverso l'attributo @sameAs.

\section{Conclusioni}

In questo contributo sono state evidenziate diverse problematiche veicolate dalla complessità dell'edizione dei Documenti d'Amore e al contempo dal campo di studio delle edizioni scientifiche digitali. L'adozione del Web semantico e dei Linked Open Data apporta ulteriori cambiamenti allo statuto epistemologico dell'editoria elettronica e digitale. Le edizioni scientifiche digitali diventano sempre più semantiche e si interconnettono con altre risorse del Web valorizzando e ampliando le pratiche che finora sono state alla base della filologia digitale. Queste sono soltanto alcune delle questioni che hanno caratterizzato il lavoro editoriale dell'opera di Francesco da Barberino. Vi sono altre importanti questioni che non sono state affrontate come per esempio l'apparato critico proposto da Antonio Montefusco e Cristiano Lorenzi. Questo perché comprenderà un secondo momento del workflow. L'infrastruttura teorica però ci permette di rendere flessibile il modello che stiamo proponendo e di ampliare aggiungendo componenti che per ragioni di tempo non sono ancora stati praticamente messi a sistema.

Il IIIF e le tecnologie per il Semantic Web ci consentono di rendere l'edizione non più un contenitore di dati scientifici che rimangono chiusi e poco accessibili ma un oggetto digitale collegato da altre risorse nel Web e potenzialmente ad altri progetti che condividono lo stesso dominio di ricerca. Ampliare il sistema informa- 
tivo a fonti esterne è uno degli obiettivi che molti progetti editoriali stanno perseguendo e riportando la discussione alla ridefinizione del significato di markup, il riutilizzo e allo scambio di dati testuali attraverso un lavoro sui testi da prospettive multiple che possono coesistere all'interno di una stessa edizione. I nuovi modelli di dati che accolgono il concetto di testo a grafo e della descrizione semantica dei dati oltrepassano ulteriormente i limiti dettati dalla codifica permettendo davvero al filologo digitale di costruire strutture semiologiche ed ermeneutiche per la critica testuale attraverso la rappresentazione digitale e le tecniche di deduzione e inferenza della formalizzazione computazionale.

\section{Bibliografia}

\section{Fonti}

Francesco da Barberino, I Documenti d'Amore secondo i manoscritti originali, I-IV, a cura di F. Egidi, Roma 1902-1927.

Francesco da Barberino, I Documenti d'Amore / Documenta Amoris, a cura di M. Albertazzi, 2 voll., Lavis $2011^{2}$ [l ed. 2008].

\section{Studi critici}

T. Berners-Lee, J. Hendler, L. Ora, The Semantic Web: A New Form of Web Content That is Meaningful to Computers Will Unleash a Revolution of New Possibilities, in «Scientific American» 5 (2001), pp. 34-43.

E. Bleeker, B. Buitendijk, R. Haentjens Dekker, Agree to disagree: Modelling co-existing scholarly perspectives on literary text, in «Digital Scholarship in the Humanities», 34/4 (December 2019), pp. 844-854, https://doi.org/10.1093/llc/fqz061.

J.D. Bolter, Lo spazio dello scrivere: computer, ipertesto e la ri-mediazione della stampa, Milano 2002.

P. Burke, Lingua, società e storia, Roma 1990.

G. Contini, Ricordo di Joseph Bédier, in Id., Esercizi di lettura sopra autori contemporanei con un'appendice su testi non contemporanei, Torino 1974.

M. Daquino, F. Giovannetti, F. Tomasi, Linked Data per le edizioni scientifiche digitali. Il workflow di pubblicazione dell'edizione semantica del quaderno di appunti di Paolo Bufalini, in «Umanistica Digitale», 7 (2019), https://doi.org/10.6092/issn.2532-8816/ 9091.

Digital philology and medieval texts, a cura di A. Ciula, F. Stella, Pisa 2007.

M. Fafinski, Facsimile narratives: Researching the past in the age of digital reproduction, in Digital Scholarship in the Humanities, 2021, https://doi.org/10.1093/llc/fqab017.

G. Genette, Palinsesti. La letteratura al secondo grado, Torino 1999. 
G. Gigliozzi, Introduzione all'uso del computer negli studi letterari, a cura di F. Ciotti, Roma 2003.

D. Goldin Folena, Il commento di Francesco da Barberino, in Intorno al testo. Tipologie del corredo esegetico e soluzioni editoriali. Atti del Convegno (Urbino, 1-3 ottobre 2001), Roma 2003, pp. 263-282.

G. Inglese, Come si legge un'edizione critica: elementi di filologia italiana, Roma 1999.

T. Mancinelli, E. Pierazzo, Che cos'è un'edizione scientifica digitale, Roma 2020.

M. Maniaci, Archeologia del manoscritto: metodi, problemi, bibliografia recente, Roma 2002.

M. McLuhan, La galassia Gutenberg: nascita dell'uomo tipografico, Roma 1984.

R. Mordenti, Appunti per una semiotica della trascrizione nella procedura ecdotica computazionale, in Studi di codifica e trattamento automatico di testi, a cura di G. Gigliozzi, Roma 1987, pp. 85-124.

R. Mordenti, L'altra critica. La nuova critica della letteratura fra studi culturali, didattica e informatica, Roma 2007.

R. Mordenti, Informatica e critica dei testi, Roma 2001.

T. Orlandi, Informatica testuale. Teoria e prassi, Roma 2010.

M.C. Panzera, Francesco da Barberino tra Andrea Cappellano e Averroè. Poesia, immagini, profetismo, Alessandria 2016.

E. Pierazzo, A rationale of digital documentary editions, in «Literary and Linguistic Computing», 26/4 (December 2011), pp. 463-477, https://doi.org/10.1093/llc/fqr033.

E. Pierazzo, Digital genetic editions: the encoding of time in manuscript transcription, in Text Editing, Print and the Digital World, London 2009, pp. 169-186.

E. Pierazzo, Digital scholarly editing: Theories, models and methods, Aldershot 2015.

E. Pierazzo, Facsimile and Document-Centric Editing, https://www.digitalmanuscripts.eu//wpcontent/uploads/2017/09/05-Digital-Facsimiles-EP.pdf.

P. Robinson, What is a Critical Digital Edition?, in «Variants: The Journal of the European Society for Textual Scholarship», 1 (2002), pp. 43-62.

P. Sahle, What is a Scholarly Digital Edition?, in E. Pierazzo, M. Driscoll (eds.), Digital Scholarly Editing: Theory, Practice and Future Perspectives, Cambridge 2015.

F. Tomasi, Digital editions as a new model of conceptual authority data, in «JLIS.IT», 4 (2013), pp. 21-44.

F. Tomasi, L'edizione digitale e la rappresentazione della conoscenza. Un esempio: Vespasiano da Bisticci e le sue lettere, in «Ecdotica», 9 (2012), pp. 264-286.

F. Tomasi, L'informazione digitale e il Web semantico. Il caso delle scholarly digital editions, in Informatica umanistica: risorse e strumenti per lo studio del lessico dei beni culturali, Firenze 2017, pp. 157-174.

F. Tomasi, F. Ciotti, M. Lana, F. Vitali, S. Peroni, D. Magro, Dialogue and linking between TEI and other semantic models, in The linked TEl: Text Encoding in the Web Roma 2013, pp. 145-158.

\section{Sitografia}

FAIR (Findability, Accessibility, Interoperability, Reuse): https://www.go-fair.org/fairprinciples; ultimo accesso: maggio 2021.

Functional Requirements for Bibliographic Records (FRBRoo): http://www.cidoc-crm.org/ frbroo/home-0; ultimo accesso: maggio 2021. 
International Image Interoperability Framework (IIIF): https://iiif.io/; ultimo accesso: maggio 2021.

Ontologia Biflow: https://catalogobiflow.vedph.it/ontology/; ultimo accesso: maggio 2021.

Text Encoding Initiative (TEI), Guidelines P5 version: https://tei-c.org/guidelines/p5/; ultimo accesso: maggio 2021. 